\section{Putting fat in its place}

\section{By Lev Osherovich, Senior Writer}

Since its discovery in 1995, VEGF-B has been overshadowed by its cousin, the proangiogenic VEGF-A, because the antiangiogenic effects of inactivating VEGF-B are modest. Now, a Swedish team has uncovered a critical biological function for VEGF-B: stimulating fatty acid transport through endothelial cells of the vasculature. ${ }^{1}$

The findings open up VEGF-B as a target for metabolic syndrome, type 2 diabetes and other indications caused by excess fatty acid accumulation. Indeed, the research has prompted CSL Ltd. to take a second look at its dormant VEGF-B mAb program and make a foray into the metabolic disease space.

Whereas VEGF-A has a well-studied role in the vascularization of tumors and diseased retinas, VEGF-B doesn't induce blood vessel formation. Instead, the growth factor is thought to help new blood vessels induced by VEGF-A

"For the first time, there
is an explanation for how
endothelial cells transfer
lipids from the environment
to target tissues."
$\quad$-Ulf Eriksson,
Karolinska Institute

larly strong in heart, skeletal muscle and brown adipose tissue and was closely correlated with the expression of genes linked to mitochondrial fatty acid oxidation.

Eriksson thus suspected that VEGF-B might upregulate mitochondrial metabolism, which relies on the oxidation of fatty acids. Indeed, cultured mouse and human endothelial cells treated with VEGF-B had higher levels of fatty acid transporter proteins than mock-treated cells. Similarly, mice overexpressing VEGF-B had more of the transporter proteins than mock-transfected controls.

To prove that VEGF-B regulates fatty acid uptake, Eriksson's team fed radiolabeled fatty acids to Vegf- $b$ knockout mice and found lower levels of radioactivity in the heart, muscle and brown adipose tissue than occurred in tissue from wild-type controls.

Eriksson thinks VEGF-B helps muscle and brown adipose tissues meet their high metabolic requirements by directing nearby endothelial cells to deliver blood-borne fatty acids. He said the process goes awry in type 2 diabetes, in which "uncoupling of fatty acid accumulation and fatty acid burning leads to increased fat accumulation in the muscles."

The results mesh with emerging evidence about how the body distributes fatty acids to various tissues, said Roger Unger, professor of internal medicine at The University of Texas Southwestern Medical Center at Dallas.

"Free fatty acids are an essential fuel, but when you have an excess of them there's nowhere for them to go" except to inappropriate tissues such as the heart and muscles, he said.

Unger noted that, consistent with Eriksson's findings, other researchers have shown that mice engineered to have myocardiocytes with increased uptake of fatty acids "develop lipidinduced apoptosis and fat accumulation in the hearts."

Blocking VEGF-B's role in cardiac fat uptake "could be very important in preventing lipotoxicity in overnourished individuals," said Unger.

He did note that lowering VEGF-B doesn't prevent fatty acid uptake by white adipose tissue, so interfering with the pathway may in fact make patients fatter. Along those lines, Eriksson's team found that Vegf$b$ knockouts gained more weight than wild-type controls fed a similar high-fat diet.

Unger recently coauthored a review article that suggested expansion of white adipose tissue, which underlies obesity, may in fact be a protective mechanism to prevent fat accumulation in the heart and liver. ${ }^{4} \mathrm{He}$ thinks white adipose tissue sequesters fat to prevent its toxic mislocalization. According to this theory, blocking transport of lipids into the heart while building up white adipose tissue could reduce complications of metabolic syndrome.

CSL's Nash noted that Vegf-b knockout mice "seem to survive quite well," suggesting that there would be few cardiovascular side effects of blocking its action. He said the company has humanized VEGF-B mAbs that are in preclinical development for metabolic indications.

Nash also said the "key experiments now underway will use models 


\section{ANALYSIS}

of type 2 diabetes to see if you can impact fat distribution and insulin sensitivity by blocking VEGF-B function."

CSL acquired a VEGF-B mAb program as part of its 2006 purchase of fellow Australian biotech Zenyth Therapeutics Ltd., which previously collaborated with Eriksson and the Ludwig Institute for Cancer Research Ltd. on uncovering VEGF-B's function. Nash said that this 10 -year-long collaboration has continued now that Zenyth is part of CSL.

Patents on the discovery are held by Eriksson's previous academic institution, the Ludwig Institute, and are licensed to CSL.

Osherovich, L. SciBX 3(13); doi:10.1038/scibx.2010.394

Published online April 1, 2010
REFERENCES

1. Hagberg, C.E. et al. Nature; published online March 14, 2010; doi:10.1038/nature08945

Contact: Ulf Eriksson, Karolinska Institute, Stockholm, Sweden e-mail: ulf.pe.eriksson@ki.se

2. Zhang, F. et al. Proc. Natl. Acad. Sci. USA 106, 6152-6157 (2009)

3. Borradaile, N.M. \& Schaffer, J.E. Curr. Hypertens. Rep. 7, 412-417 (2005)

4. Unger, R.H. \& Scherer, P.E. Trends Endocrinol. Metab.; published online Feb. 23, 2010; doi:10.1016/j.tem.2010.01.009

COMPANIES AND INSTITUTIONS MENTIONED

CSL Ltd. (ASX:CSL), Melbourne, Victoria, Australia Karolinska Institute, Stockholm, Sweden Ludwig Institute for Cancer Research Ltd., New York, N.Y. The University of Texas Southwestern Medical Center at Dallas, Dallas, Texas 